(Aus dem anatomischen Institut in Strasshurg.)

\title{
Idiozom und Centralkörper im Ovarialeie der Säugethiere.
}

\author{
Von
}

Dr. Alexander Ginrwitseh,

Assistent am anatomischen Institut.

Hierzu Tafel XVI.

Bei einer Untersuchung von Ovarien neugeborener und etwa 12 täigiger Meerschweinchen, eines für das Studium der s. g. Dotterkerne ausserordentlich günstigen Objektes, ist es mir gelungen, in den Dotterkernen die bis jetzt meines Wissens noch stets vermissten Centralkörper mit aller erwünschter Deutlichkeit zur Darstellung zu bringen. Hält man sich an ein junges, 12 tägiges Thier, in dessen Ovarium die Theilungsperiode schon vollständig abgelaufen ist, so kann man das Verhalten des völligr ausgebildeten Dotterkernes und in den älteren, von einer 2-3 reihigen Zona granularis umgebenen Eiern, anch das Zugrundegehen desselben studiren; ein kaum ausgetragenes Thier liefert dagegen die erwünschte Ergünzung zu den Bildern des ruhenden Dotterkernes: die meisten Eier befinden sich daselbst in verschiedenen Theilungsstadien und es gelingt mit Leichtigkeit, das Verhalten des Dotterkernes und der darin eingeschlossenen Centralkörper bei der Mitose zu verfolgen.

Ich fange mit der Beschreibung der auf der Höhe ihrer Ausbildung stehenden Dotterkerne an: sie sind am schönsten bei jungen, schnell wachsenden Eiern zu beobachten, welche schon von einer einreihigen Zona granularis umgeben sind: der sehr grosse kugelige Eikern liegt meistens etwas excentrisch, der Eiperipherie genähert: das Chromatin ist in einem sehr lockeren, weitmaschigen Netze angeordnet, häufig annähernd radiär um einen nucleolusähnlichen Chromatinklumpen gelagert (Fig. 1). Das Cytoplasma ist hei der angewandten Fixirung (Carnoy's Gemisch 
18-24 Stunden) sehr locker gebant: es zeigt einen bald mehr reticulären, bald faserigen Bau: es sind hie und da auch dickere Faden eingelagert, welche sich mit Vorliebe um den Kern herum concentrisch anordnen: ziemlich zahlreich sind auch körnige Plasmaeinlagerungen: es sind kleine Kugeln von sehr verschiedener Grösse: sehr häufig scheinen 2 bis 3 grössere Substanzanhäufungen zu sein, welche sich ziemlich intensiv mit Eisenhaematoxylin färben und vielleicht den s. g. chromatoiden Körpern der Spermatocyten entsprechen (s. v. Lenhossek, 6).

Der Dotterkern ist in jeder Eizelle voll ausgebildet vorhanden: er stellt einen runden, zuweilen auch länglichen Körper dar, welcher etwa die Grösse eines rothen Blutkörperchens erreicht. Seine Conturen sind in den älteren Eiern sehr scharf gegen das angrenzende Cytoplasma abgesetzt: nur selten sind sie von einer unregelmässigen gelappten Form, wie Meves es für die Nebenkerne in den Samenzellen des Salamanders abbildet (Fig. 3). Dic Substanz des Nebenkernes hat ein völlig homogenes Aussehen und es ist mir nie gelungen, eine radiäre oder concentrische Streifung derselben nachzuweisen: ein sehr häufiger, ja fast regelmässigcr Befund ist dagegen ein heller Ring, welcher die dunkler gefärbte Substauz in eine scheibenförmige Innenzone und einen Aussenstreifen sondert (Fig. 1, 2, 3); auch dieses Bild erinnert lebhatt an die von Meves für den Salamanderhoden gegebenen Bilder (7, Fig. 40,41). Der Dotterkern sticht bei jeder Färbung sebr dentlich vom umgebenden Cytoplasma ab: amschönsten sind jedoch die mit Eisenhaematoxylin und Rubin behandelten Präparate: an denselben ist der Dotterkern das einzige rosaroth gefärbte Gebilde der Eizelle: sowohl der Eikern als das Cytoplasma sammt allen Einschlüssen behalten die grauschwarze Farbe des Eisenhaematoxylins; auch die chromatoiden Nebenkörper sind schwarz gefärbt. Es hat schon Lenhóssek (6) für die Spernatocyten der Ratte richtig hervorgeboben, dass an mit Eisenhaematoxylin überfärbten oder nicht genügend differenzirten Präparaten der Nebenkern dennoch nicht schwarz gefärbt ist, sondern höchstens schwarze Niederschläge auf seiner Oberfläche zeigt, durch welche die bei einer Nachfärbung hellroth gefärbte Substanz des Nebenkernes deutlich durchschimmert. Dasselbe trifft in vollem Maasse auch für die Dotterkerne unseres Objektes za. Eine Spiegelfärbung im 
Sinne A. Fischers (4) konnte ich trotz verschiedener Abstufungen die Differenzirung nie erhalten.

Im Centrum der Dotterkerne lassen sich nun mit der grössten Schärfe Centralkörper nachweisen: dieselben sind gewöhnlich in der Zweizahl vorhanden und dicht beieinander liegend, in vielen Fällen kann ein dünner Verbindungsstreifen zwischen denselben - eine Centrodesmose Heidenhains - wahrgenommen werden (Fig. 3): zuweilen sieht aber umgekehrt der Zwischenraum wie eine sehr kleine helle Spindel aus, an dessen Polen die Centralkörper sitzen (Fig. 2). Ziemlich häufig ist auch das Vorkonmen von zwei ilantelförmigen Centralkörpern-Diplosomen (Fig. 3). Von ciner bestimmten festen Orientirung der Centralkörperachse zum Zellkern oder zur Zellachse konnte ich mich nicht überzeugen: cine zum Kerne tangentiale Richtung der Centralkörperachse scheint ebenso häufig wie eine normale oder schiefe $z u$ sein.

Es ist schwer, mit Bestimmtheit den Zeitpunkt des Schwindens des Dotterkernes in den Eiern zu bestimmen. Bei den von einer 2-3 reihigen Zona granularis umgebenen Eiern, dic zn den ältesten des Ovaris eines 12 tägigen Thieres gehören, babe ich in der Mehrzahl keinen deutlichen Dotterkern finden künnen, wohl aber einzelne roth gefärbte Substanzbrocken, welche vielleicht als Zerfallprodukte desselben angesehen werden diuften. In den wenigen Fällen, wo ein noch einheitlicber Dotterkern zu beobachten war, hatte er stets eine sehr excentrische Lage; die Conturen waren unregelmässig, wie geschrumpft; Centralkörper liessen sich in solchen degenerirenden Dotterkernen nie mit Sicherheit nachweisen. Ich muss daher auch vorläufig die Frage offen lasseu, ob in einem reifen $\mathrm{Ei}$, nachdem es des Dotterkernes verlustig wurde, noch die Centralkörper persistiren und eventuell bei der Richtungsspindel eine Rolle spielen: dass man bei pathologischen Mitosen, welche so häufig in den atretischen Follikeln beim Meerschweinchen auftreten und zum Theil wohl als echte Furchungen angesehen werden dürten, zuweilen an den Spindelpolen schwarze Körnchen auftreten sieht, die sich in keiner Hinsicht von den Centralkörpern unterscheiden, wurde u. A. dureh Rabl's (15) Untersuchungen bekannt und kann auch an dieser Stelle von mir bestätigt werden. Von der Mehrzahl der Autoren wurden jedoch Centralkörper in den echten normalen Richtungsspindeln der Sängethiereier vermisst (Sobotta 16, 17). 
Ein einwandsfreier Nachweis von nackten, von keiner typischen Sphäre umgebenen Centralkörpern innerhalb des Eicytoplasmas, nachdem der so leicht zu erkennende Dotterkern verschwunden ist, dürfte wohl schwer gelingen: das Eiplasma ist nämlich so reich an verschiedenen mikrosomalen Einschlüssen, dass ein schwarzes Doppelkörnchen, welches ja ab und $\mathrm{zu}$ in demselben auftritt, nie eine sichere Deutung seiner Natur erfahren kanu.

Es ist nun von grossem Interesse, das Verhalten der Dotterkerne sammt den in ihnen eingeschlossenen Centralkörpern bei der Mitose zu verfolgen. Es dürften meines Erachtens nur diejenigen centralkörperällnlichen Gebilde für wirkliche Centralkörper erklärt werden, welche nachgewiesenermaassen bei der Mitose eine entsprechende Function übernehmen. Dieser Beweis ist für viele Fälle noch nicht erbracht, so. \%. B. für die s. g. Centralkörper der Cylinderepithelien, den Ganglienzellen etc.

Bei dem uns an dieser Stelle interessirenden Objekt sind wir in einer guinstigeren Lage, wie aus den Bildern Fig. 4-8 zu ersehen ist.

Die betreffenden Abbildungen sind einem Ovar eines fast völlig ausgetragenen Meerschweinchens entnommen. Die meisten Ovocyten befinden sich in reger Theilung, wobei ganz auffallend das Knäuelstadium vorherrscht. Der Dotterkern ist bald mehr bald weniger scharf von den umgebenden Cytoplasma abgegrenzt, in sehr vielen Fällen ist die Conturirung desselben eine ebenso präcise, wie bei den älteren in Figur 1-3 abgebildeten Eiern (Fig. 4), der Centralkörper innerhalb des Dotterkernes besteht aus einem typischen Doppelkorn, häufig mit einer deutlichen Centrodesmose.

Bei dem weiteren Fortschreiten der Mitose lagert sich der Dotterkern dem Kerne dichter an, verliert seine schafe Contur, die Substanz des Dotterkernes zerfliesst gewissermaassen an der Oberfläche des.Kernes, sticht aber noch immerhin sehr deutlich von dem anliegenden Cytoplasma ab. Ganz analoge Bilder sind uns schon bei den Mitosen der Spermatocyten beim Salamander durch Meves bekannt geworden (Fig. 5, 6). Gleichzeitig mit dem Zerfliessen des Dotterkernes tritt zwischen den beiden, etwas auseinanderweichenden Centralkörpern eine helle spindelförmige Stelle auf, anscheinend die erste Anlage der Centralspindel; bei 
der relativen Kleinheit der Verhältnisse ist diesclbe nicht immer ohne weiteres als solche zu erkennen, namentlich wo keine Spur einer Strahlung wahrzunehmen ist. Das sehr häufige Vorkommen des Bildes und namentlich die weiter zu beschreibenden Stadien schliessen jedoch jeden Zweifel aus, dass wir es wirklich mit einer Centralspindel zu thun haben, wir brauchen nur die Fig. 7 einer Betrachtung zu unterziehen. Die Kernmembran ist nun geschwunden, der Knänel in einzelne Segmente zerfallen, welche sich in einen Mutterstern ordnen. Im Centrum desselben ist eine sehr deutliche Centralspindel zu sehen, an dessen Folen Centralkörper sitzen und eine deutliche Strablung ausgeht.

Das Bild ist aus den zahlreichen Darstellungen der Mitose so bekannt, dass es kaum einer näheren Erläuterung bedarf.

Es ist aber insofern von grosser Bedeutung, als es die Deutuny. der innerhalb des Dotterkernes bei der Eisenhiematoxylinfärbungr auftretenden schwarzen Körnchen als wahre Centralkörper ganz sicher stellt.

Wir können somit mit gutem Rechte den Dotterkern der unreifen Sä unethiereier dem Idiozom der Samenzellen rollständig homologisiren. Sowohl die männlichen als die weiblichen Genitalzellen haben somit die Eigenthümlichkeit, dass die Centralkörper derselben von einer deutlich differenzirten, anscheinend in ihrer Zusammensetzung sehr constanten und von dem umgebenden Cytoplasma scharf abgegrenzten Hülle ungeben sind.

Es ist schon vielfach der Versuch gemacht worden, die Dotterkerne des Eies dem Nebenkerne der Samenzellen zu homo. logisiren. Die Aehnlichkeit beider Gebilde ist in der That in manchen Fällen eine ganz auffällige, da jedoch einige wichtige Punkte im Aufbau (namentlich der bis jetzt noch nicht dargebrachte Nachweis von Centralkörpern in den Dotterkernen) und das Endschicksal beider in Frage kommenden Bildungen stark differiren, ist auch diese Deutung vorlänfig über das Stadium der Wahrscheinlichkeit nicht hinausgekommen und hat neben zahlreichen Verfechtern auch entschiedene Gegner aufzuweisen.

Henneguy, welcher Ovarialeier zahlreicher Repräsantanten von Wirbelthieren und namentlich von Säugern untersuchte, sieht im Dotterkern „un organe ancestral qui avec les élements nucléo- 
laires de la vesicule germinative correspond au makronucleus des Infusories, le mikronucleus étant représanté par le reseau chromatique, prenant seul part aux phénomènes de fécondation" (5, S. 35).

Wenn auch diese Definition etwas unbestimmt lautet, so ist um desto entschiedener die Stellungnahme Balbi an i's (2): er sieht im Dotterkern der Araneiden ein Homologon des "Centrosoma" im Sinne B overi's, d. b. eine Attractionssphäre sammt dem darin eingeschlossenen Centralkörper, ohne allerdings wirkliche Centralkörper oder das Verhalten des Dotterkernes in der Mitose gesehen zu haben. In einer fast gleichzeitig mit der obengenannten erschienenen Arbeit tritt M ertens (10) der Deutung Balbiani's entschieden entgegen; nach diesem Autor sollen unter dem Namen "Dotterkern" zwei grund verschiedene Bildungen beschrieben worden sein, bald sind es Attractionssphären, welche schon den Primordialeiern zukommen, bei denen Mitosen thätig sind und noch eine Zeitlang bei den sich nicht mehr theilenden individualisirten Eiem in der Wachsthumsperiode persistiren (wobei jedoch Centralkörper in den ruhenden Eiern nur a as a hoswe isc nachzuweisen wären), es sind somit Bildungen, welche mit den von den Autoren, z. B. H e n n e g u y, beschriebenen Dotterkernen nichts gemeinsam haben, da ja letztere, nach Henneguy's Angaben, in den Primordialeiern noch fehlen und erst in der Wachsthumsperiode auftreten sollen.

Die von Henneguy beschriebenen, mit Safranin sich roth färbenden Kugeln sind dagegen nach $\mathrm{Mertens}$ nucleoläre Produkte, die aus dem Kern in das Eiplasma ausgestossen werden; dass dieselben jedoch nicht centrosomaler Natur sind, erhellt nach Mertens aus ihrem Vorkommen in den Eizellen gleichzeitig mit echten Attractionssphären, ja sogar in mitotisch sich theilenden Primordialeiern: in letsteren Fällen sind an den Spindelpolen deutliche Centralkörper sichtbar, im Eiplasma liegen dagegen die Körner, welche nach Mertens' Meinung von Henneguy für Dotterkerne angesprochen wurden.

Die Angaben Balbiani's bleiben durch Mertens Behanptungen zunächst unberührt, es ist auch sehr fraglich, ob die safranophilen Körner, die von ihm in den Eizellen neben den ${ }_{n}$ Sphères attractives" beobachtet wurden, wirklich mit den Henneguy. 
schen Dotterkernen identisch sind - ich glaube vielmehr, dass es sich um die oben erwähnten chromatoiden Körper handelt.

Ist aber II e r tens letzte Behauptung nicht stichhaltig, so wendet sich M e r te n s Waffe gegen ihn selbst.

Die zwei neuesten Publicationen über (len Dotterkern rülren von O. van der Stricht (18) und van Bambecke (3).

Nachdem Eisterer die Genese des Dotterkernes in den Ovocyten des Weibes and der Spinne aus der s. g. "Couche vitellogène ${ }^{\prime}$ besprochen hat, wendet er sich zur Frage über die Bedeutung des Dotterkernes: er neigt zu der oben citirten Ansicht von Balbiani, hält aber die Frage trotzdem nicht für spruchreif :

${ }_{n}$ Pour établir avee tout la certitude voulue cette homologie (se. mit dem Nebenkern der Samenzellen) . . . il fandrait ou bien pouvoir établir que le noyau vitellin provient de la sphère attractive persistant après la dernière division des oogonies ... ou bien qu'elle engendrera la sphère attractive du premier fuseau de maturation ${ }^{\prime}$ (S. 138). Letzterer Nachweis wird meines Erachtens nach dem oben Auseinandergesetyten kaum gelingen, ersteren glaube ich an dieser Stelle dargebracht $z$ u baben.

v. B a mbecke (3) hält den Dotterkern für cinen „Centre des formations plastiques de vitellus", eine Deutung, die von der unserigen anscheinend abweichend ist. Indem Me ve s (8) statt der wenig präcisen und von verschiedenen Autoren selur widersprechend gebrauchten Bezeichnung ${ }_{n}$ Sphäre $^{\text {" }}$ für die Nebenkerne der Samenzellen den Namen „Idiozom " vorschlug und zugleich eine Characteristik derselben gah, versuchte er dem neugeschaffenen Begriffe auch die Dotterkerne der Eier zu subsummiren: „die gleiche Bezeichnung (Idiozom) würde auch auf die s. g. Dotterkerne der Ovarialeier, welche nach Flemming und Balbiani als Homologa der in den Hodenzellen auftretenden Gebilde aufzufassen sind, Anwendung finden müssen, obgleich der sichere Nachweis von Centralkörpern in ihnen meines Wissens noch nicht erbracht ist" (S. 318). Ich glaube aber, dass in diesem Nachweis der Kernpunkt der Frage liegt: dass das Endschicksal der beiden in Betracht kommenden Gebilde ein ganz verschiedenes ist, wurde durch die spermatologischen Untersuchungen der letzten Jahre mit aller Dentlichkeit dargethan.

Eine Gleichstellung des Dotterkernes mit dem Nebenkern 
kann daher nur auf Grond einer Uebereinstimmung in der Entstehung und event. anch im Bau der fertigen Gebilde versucht werden.

Ich glaube daher, dass erst durch den sicheren Nachweis von Centralkörpern innerhalb der Dotterkerne die Homologisirung beider Gebilde vollständig berechtigt wird, namentlich, wenn man, wie ich es zu thun geneigt wäre, das Hauptgewicht bei der Definition des Idiozoms auf die $\mathrm{Anw}$ es e $\mathrm{n}$ he it von $\mathrm{C}$ en. tralkörpern innerbalb einer "specifisch beschaffenen Hülle" (M e ves) legt.

Es wäre nun noch genauer zu untersuchen, wie die Dotterkernsubstanz, also das Idiozom des Eies, sich bei der Mitose verhält (s. o. S. 381). Es sind daruber bis jetzt keine genaueren Angaben vorhanden, da die Mehrzahl der Autoren den Dotterkern in den Eiern der Wachsthunsperiode untersueht haben, bei welchen vielleicht $a b$ und zu eine Mitose eintreten dürfte, im ganzen aber zur Seltenheit gehört.

M e r t e n s (10) bildet eine Mitose eines Primordialeies ab, in welchem jedoch die Substanz seiner "S̄phère attractive" noch nicht scharf individualisirt ist: es kam das Stadium des Muttersternes zür Abbildung, an dessen beiden Polen, um die Centralkörper herum, eine Anhänfung von dichterem Protoplasma zu sehen ist.

Desto zahlreicher sind die Angaben uber die Rolle des Idiozoms in den Mitosen der Spermatocyten: Der Vorgang scheint nach mehreren verschiedenen Typen zu verlaufen, wie es auch u. A. Meres in seinem letzten Bericht (9) bethont.

Bei den Spermatocythen des Salamanders wird nach den Angaben von Meves durch die heranwachsende, im Idiozom entstehende Centralspindel ersteres gesprengt; die einzelnen Brocken der Idiozomsubstanz lagern sich dem Kern dicht an, flachen sich $a b$ und werden allmählich der Beobachtung entzogen. Durch den beschriebenen Vorgang frei gewordene Centralspindel kann nunmehr zur Bildung der achromatischen Theilungsfigur in directe Verbindung mit den Fäden der Zellsubstanz treten.

Die in manchen anderen Punkten abweichende Angaben von Rawit\% (14) stimmen in der uns beschäftigenden Frage mit denen von Meves iiberein, indem er ebenfalls von einer Zerbröckelung der Idiozomsubstanz spricht. Aehnlich lauten die 
Angaben von M u r r a y über die Fragmentirung des Nebenkernes bei der Mitose der Spermatocyten der Helix (12).

Ganz anders scheint der Vorgang bei den Spermatocyten der Ratte nach den Angaben von $L$ e n hós ek zu verlaufen (6): Die Centralkörper wandern sehr frühzeitig aus dem Nebenkern heraus, wobei letzterer zunächst intact bleibt und nur eine Schrumpfung erleidet. Ob und wann zwischen den Centralkörpern eine Centralspindel auftritt ist aus v. L e n hossék's Angaben nicht zu ersehen. Im Stadium des Muttersternes soll jedoch die Sphäre noch deutlich als ein geschrumpfter, in der Peripherie der Zelle gelegener Klumpen zu sehen sein.

Es wäre noch der Angaben von Mo o re (11) zu gedenken, welcher die Centralspindel in den Spermatocyten der Elasmobranchier aus der Substanz des Nebenkerns hervorgehen lïsst.

Meine Befunde an dem Idiozom des Meerschweincheneies knüpfen sich am engsten den letrterwähnten Angaben an. Im Starlium eines lockeren Knänels ist bei den jungen Ovogonien das Idio\%om in seiner vollen Integrität noch zuweilen vorhanden (Fig. 6). Die beiden Centralkörper sind bald durch einc Centrodesmose, bald durch eine helle Stelle, welche als die erste Anlage der Centralspindel angesprochen werden dürfte, verbunden.

Im weiteren Fortschreiten der vorbercitenden Mitosenstadien kann ich eher von einem allmählichen Zerfliessen der Idiozomsubstanz, als von einer Zerbröckelung derselben sprechen (Fig. 5): Man sieht dem Kerne an einer Stelle eine riemlich dentlich abgegrenzte, im Gegensatz zu dem iubrigen Cytoplasma leuchtend roth (bei Rubin-Nachfärbung) gefärbte Sichel angelagert, in deren Innerem eine kleine Centralspindel sehr deutlich hervortritt.

Von einer Strahlung innerhalb der Idiozomsubstanz oder des Cytoplasma ist noch nichts wahrzunehmen.

Bestimmend für die Deutung der weiteren Rolle des Idiozoms scheint mir aber das oben erwähnte in Fig. 7 abgebildete Stadium der Mitose zu sein: Im Centrum der in einem Kreise gelagerten Chromatinschleifen ist in einer compacten roth gefürbten Substanz die Centralspindel eingebettet; von beiden Polen derselben geht eine dentliche Strahlung aus, die sich zu den Chromosomen zieht: Es kann somit gar keinem Zweifel unterliegen, dass bis in die späteren Stadien hinein, die achromatische 
Figur in der Substanz des Idiozoms liegen bleibt und von derselben nicht scharf zu trennen ist. Ob man berechtigt ist, daraus einen Schluss auf die Herkunft der ganzen Strahlung einer völlig ausgebildeten Mitose aus der Idiozomsubstanz zu ziehen, ist eine andere Frage, die ich hier nicht entscheiden kann. So viel kann ich aber behaupten, dass wälirend der weiteren Ausbildung der achromatischen Figur, die Idiozomsubstanz nur ganz allmählich schwindet, so dass z. B. an den Polen einer Muttersternfigur die grauschwarz gefürbten, gegen den Centralkörper za convergirenden Spindelfasern noch von Spuren derselben umgeben sind. Andererseits konnte ich ausser der Anhäufung der Idiozomsubstanz um die Centralspindel nie andere Substanzbrocken oder sonstige Residuen des Idiozoms bei den versehiedenen Mitosestadien finden.

Es wäre gewagt, in anbetracht der scharf umgrenzten Rubinfärbung des Idiozoms, von einer specif is chen fürberischen Reaction der Substanz derselben zu sprechen und jede, entsprechend gefärbte Substanzanhäufung im Eicytoplasma für einen Bestandtheil oder Product des Idiozoms zu erklären.

Etwas anderes ist es aber, wenn man den ständigen, in allen Mitosestadien bewahrten Zusammenhang der distinct rothgefürbten Substanz mit den Centralkörpern resp. der Spindel beobachten kann, wie es in unserem Falle bei der Mitose der Eizellen der Fall ist. Die Unterschiede der beiden benachbarten Stadien Fig. 6 und 7 sind so graduell, dass man mit Sicherheit behaupten kann, dass die zunächst noch sehr begrenzte in Fig. 7 dargestellte Strahlung durch entsprechende Umformung der Idiozomsubstanz der Fig. 6 entsteht. Diese Gestaltänderung könnte vielleicht auf einer einfachen Modification des Aggregatzustandes der Idiozomsubstanz beruhen: Würde dieselbe z. B. flüssiger werden, so könnte bei ihrem Zerfliessen im umgebenden Cytoplasma die Strablung auf einem von A. Fischer(4) angegebenen Wege zu Stande kommen.

Damit will ich natürlich nicht gesagt haben, dass man sich die Ursache des Auftretens der Strahlung auf diesem Stadium nicht auch anders vorstellen könnte. Je weiter die achromatische Figur ausgebildet wird, desto mehr schwindet die Anhäufung der Idiozomsubstanz um die Fasern der ersteren. Die achro. matische Figur beschränkt sich fast ausschliesslich auf eine 
Centralspindel und halb durehgehende Fasern. Von einer Polstrahlung sind nur in vereinzelten Fällen schwache Andeutungen wahrnehmbar (Fig. 8). Wie ich schon oben hervorgehoben habe, lassen sich über das Material für die ganze definitive achromatische Figur nur mehr oder weniger begründete Vermuthungen aussprechen: Es läge am Nächsten, dieselbe in ihrem ganzen Umfange aus der Idiozomsubstanz abzuleiten. Es spricht dafiir ausser den oben angefühtten Gründen noch der folgende Umstand: Die Idiozomsubstanz ist trotz ihrer sehr scharfen Abgrenzung von dem umgebenden Cytoplasma wachstlums- und folglich auch assimilationsfähig: Man kann es aus der regelmässigen, sehr bedentenden Grössenzunahme des Idiozoms der älteren Eier (Fig. 1-3) im Vergleich 7 demjenigen der jungen $(4,5,6)$ ersehen: Es lässt sich somit leicht denken, dass, indem die ursprüngliche Idiozomsubstanz zur Bildung der auftretenden Strahlung verbrancht wirl, immer nenes Cytoplasma in den Bereich derselben aufgenommen wird und auf diesem Wege das Material fiir die ganze achromatische Figur liefert.

Es wäre auch an eine nucleäre Herkunft eines Theiles der achromatischen Figur, namentlich der Insertionsfasern derselben zu denken: Eine solche wurde u. A. in der letzten Zeit von $\mathrm{Ni}$ essing (13) bei Spermatocyten des Salamanders beschrieben, ich konnte jedoch in meinen Prïparaten keinen Anbaltspunkt fiir eine derartige Annahme finden.

Eine Verwendung der Idiozomsubstanz für die Bildung der Strahlenfigur wurde u. A. von Auerbach für die Spermatogonien der Paludina vivipara beschrieben (1): Der in einen Meniscus abgeflachte Nebenkern theilt sich auf einem bestimmten Mitosestadium in zwei Portionen, die sich an die entgegengesetzten Pole der Zelle begeben und aus sich die Strahlung der Spindel hervorgehen lassen. Auch für die grossen Spermatogonien des Salamanders wird ein ähnliches Verhalten der Idiozomsubstanz von Meves (8) angenommen: „Man könnte . . . solange entgegengesetzte Beobachtungen nicht vorliegen, muthmassen, dass auch in diesen Zellen, anders wie in den Spermatocyten desselben Thieres, die Masse des Idiozoms zum Aufbau der Spindelfasern im Beginn der Mitose herangezogen wird."

Me ves kommt auf diese Möglichkeit auf Grund der Thatsache, dass das Idiozom nach Ablauf der Mitose sich aus 
den Spindelfasern, oder einer in ihnen enthaltenen Substanz reconstruirt. El glaubt wenigstens, seine Bilder nur in diesem Sinne deuten zu können.

Es wäre sebr interessant, wenn es sich bestätigen liesse, dass die ältere, d. h. in den älteren Samenzellen (Spermatocyten) vorhandene Idiozomsubstanz ein anderes Verhalten, vielleicht in Folge einer verïnderten Consistenz, als in den jüngeren Samenzellen, den Spermatogonien, aufweist.

Nach den von mir in den jungen und äteren Ovarialeiern beobachteten Bildern ist es nämlich für mich im hohen Grade wahrscheinlich, dass die Consistenz der Idiozomsubstanz in den älteren, in der Wachsthumsperiode stehenden Eiern eine ganz andere, und zwar eine viel compactere, als bei den jungen sich theilenden Eiern ist.

Ich habe in den Fig. 5 u. 7 ein allmähliches Zerfliessen der Idiozomsubstanz obne scharfe Grenzen gegen das Eiplasma beschrieben. In den älteren Eiern ist es dagegen nichts Seltenes, einzelne eckige Defecte in den scharf abgesetzten Conturen der Idiozomen zu treffen (Fig. 2). Man hat den Eindruck, als ob sich die Idiozomsubstanz zerbröckeln liesse.

Ein ähnliches Verhalten, wie das letzterwähnte Bild zeigen ja ubrigens die Abbildungen mehrer Autoren, in besonders exquisiter Weise bei $\mathrm{M} u$ rra y (12). Ob es sich wirklich ähnlich auch im lebenden Objecte verhält, könnte natürlich nur aus einer Prüfung des Lebenden erschlossen werden. Letztere dürfte sich auch leicht bewerkstelligen lassen. Ich konnte sie aber leider vorläufig nicht vornehmen, da mir für die vorliegende Untersuchung nur conservirtes Material zur Verfuggung stand.

Der Vorgang der Reconstruction des Idiozoms nach dem Ablauf der Mitose wurde meines Wissens bis jetzt noch nicht genügend verfolgt.

Me ves (7) nimmt für die Spermatogonien des Salamanders an, dass das Idiozom nach Ablauf der Mitose sich aus den Spindelfasern oder einer in ihnen enthaltenen Substanz reconstruirt; nach der ersten Reifungstheilung der Spermatocyten kommt es dagegen nicht mehr zu einer Bildung eines einheitlichen compacten Idiozoms, man sieht vielmehr in der Umgebung des Centralkörpers einzelne Brocken der Idiozomsubstanz liegen. Bei Arion lässt sich dagegen nach Murray (12) mit Sicherheit 
feststellen, dass die Idiozomfragmente in den Spermatocyten zweiter Ordnung wieder zu einem einheitlichen Körper verschmelzen.

Bei den Spermatocyten der Ratte entziehen sich nach Len hóssek (6) nach dem Stadium der Metakinese die Nebenkernreste dem Blicke. Auf welche Weise der neue Nebenkern in den Tochterzellen sich reconstruirt, konnte v. Le n hós sek nicht mit Sicherheit nachweisen. Dieselbe Lücke in der Verfolgung des Idiozoms muss ich leider auch für mein Object zugeben. In den Schlussfasen der Mitose wird die ganze achromatische Figur auf ein Minimum reducirt: Es bleibt nur der compacte Spindelrest mit dem Zwischenkörperchen übrig, von Spindeln oder Strahlung an den gewesenen Polen der Mitose, in der Umgebung der Centralkörper, ist dagegen keine Spur mehr vorbanden. Ebenso wenig liess sich in meinen Präparaten irsend eine Substanzanhäufung um dic Centralkörper nachweisen.

Es ist mir somit völlig unklar geblieben, wie die nachträigliche Anhäufung der Idiozomsubstanz, um die Centralkörper zu Stande kommt. Es liegt wohl die Vermuthung nahe, dass die Substanz der Spindel, die sich in den Telophasen im Cytoplasma gelöst hat, nachtrüglich wieder um den Centralkörper als Centrum "herauskrystallisirt." Beweisen lïsst es sich aber keinesfalls; ich möchte jedoch nur noch erwähnen, dass der Spindelrest mit dem Zwischenkürperchen sehr leblıaft Rubin aufnimmt und darin an die Idiozomsubstanz erinnert. Ich glaube abel kaum, dass man diesem färberischen Verhalten einen zu grossen Werth beimessen kann.

Ich glaube somit, dass man aus dem Vorhergehenden folgende Thatsachen als sicher festgestellt annehmen kann:

1. Der s. g. Dotterkern der jungen, sich theilenden Orocyten ist eine wirkliche Sphäre im Sinne r. Benedens, d. h. besteht aus einer Anhäufung einer Plasmamasse, welche gegen das nmgebende Cytoplasma scharf abgegrenzt ist und die Centralkörper umbüllt: Bei der Mitose entstebt aus dieser Plasmaanhafung sicher ein Theil, wahrscheinlich a ch die ganze achromatische Figur.

Die scharfe Abgrenzung dieser Plasmamasse von dem umArch. f. mikrosk. Anat. Bd. 56 
gebenden Plasma berechtigt uns auch zu der von M e ves für die Nebenkerne der Samenzellen eingeführten Bezeichnung "I d i o z o m".

$\mathrm{Ob}$ dieser Idiozomsubstanz eine specifische $\mathrm{ch}$ e mi s $\mathrm{che}$ Beschaffenheit, oder nur ein anderer Aggregatzustand, als dem umgebenden Cytoplasma zukommt, lässt sich nicht sicher feststellen.

2. Nachdem das Idiozom der Ovocyten im Laufe der Mitose vollständig aufgebraucht wurde, tritt es in den Eiern der Wachsthumsperiode wiederum als eine deutlich abgegrenzte Hülle der Centralkörper auf: es scheint dabei seine physikalische Beschaffenheit eine etwas andere und zwar cine consistentere geworden zu sein, indem die Idiozomsubstanz sich unter Umständen zerbröckelt, und auch unter diesen Erscheinungen in den älteren Eiern zu Grunde geht, resp. sich der Beobachtung entzieht. Auch in dieser Modification ihrer Beschaffenheit in den älteren Eiern, zeigt die Idiozomsubstanz des Eies dic grösste Aehnlichkeit mit derjenigen der Samenzellen, indem sie sich bei den Spermatogonien des Salamanders anders, als bei den Spermatocyten verhält (M e ves 7).

3. Eine directe Abstammung dieser späteren Idiozomsubstanz aus der bei den Mitosen der Ovocyten thätigen, lïsst sich, an unserem Objecte wenigstens, nicht sicher nachweisen, jedoch mit Wahrscheinlichkeit vermuthen.

Strassburg i. E. d. 17. März 1900.

\section{Literatur-Verzeichniss.}

1. Auerbach, Untersuchungen über die Spermatogenese von Paludina Vivipara. Jenaische Zeitschrift f. Naturw. Bd. 30. N. F. 23. 1896.

2. Balbiani, Centrosome et "Dotterkern“. Journal d'Anatomie. 1893.

3. Bambecke, C. van, Archives de Biologie. 1898.

4. A. Fischer, Fixirung, Färbung und Ball des Protoplasmas. Jena 1899.

5. Henneguy, Le corps vitellin de Balbiani dans L'oeuf des Vertébrès. Journal d'Ánatomie. 1893.

6. v. Lenhóssek, Untersuchungen über Spermatogenese. Archiv f. mikroskopische Anatomie Bd. 51. 1898.

7. Meves, F., Ueber die Entwickelung der männlichen Geschlechts- 
Idiozom und Centralkörper im Ovarialeie der Säugethiere.

zellen von Salamandra maculosa. Archiv f. mikroskopische Anat. Bd. 48. 1897.

8. Derselbe, Ergebnisse von Meckel und Bonnet Bd. 6. 1896.

9. Derselbe, Ergebnisse von Meckel und Bonnet Bd. 8. 1898.

10. Mertens, Recherches sur la signification du corps vitellin. Arch. de Biologie Bd. XIII. 1893.

11. Moore, On the structural changes in the reproductive cells during the spermatogenesis of Elasmobranche. Quart. Journ. of microse. Science. V. 38. 1895.

12. Murray, Contributions to a knowledge of the Nebenkern in the Spermatogenesis of Pulmonata - Helix et Arion. Zoologische Jahrbücher. XI. 1898.

13. Niessing, G., Zellstudien. Archiv f. mikrosk. Anat. Bd. 55. 1899.

14. R awitz, Untersuchungen über die Zelltheilung. Archiv f. mikrosk. Anatomie Bd. 53. 1898.

15. Ra bl, H., Zur Kenntniss der Richtungsspindeln etc. Sitzungsber. der Wiener Akademie Bd. 106.

16. Sobotta, Die Befruchtung und Furchung des Eies der Maus. Archiv f. mikroskopische Anatomie Bd. 45. 1895.

17. Derselbe, Festschrift des medicinisch naturwiss. Vereins in Würzburg. 1899.

18. van der Stricht, Contribution à l'étude du noyau vitellin de Balbiani dans l'oocyte de la femme. Verhandlungen der anatomischen Gesellschaft in Kiel. 1898.

\section{Erklärung der Figuren anf Tafel XVI.}

Sämmtliche Figuren von Meerschweinchenovarien. Fixirung in Alkohol-Eisessig-Chloroform. Fürbung mit Eisenhämatoxylin-Rubin. Vergrösserung: Seibert Apochromat $2 \mathrm{~mm}$. Comp.Ocular 12. Hühe des Objecttisches.

Fig. 1. Ein Ei aus der Wachsthumsperiode: Ovar eines 12 tïgigen Thieres. 1-2 reihige Zona granularis. Idiozom mit 2 Centralkörpern. Im Eiplasma zwei chromatoide Nebenkörper.

Fig. 2 u. 3. Abweichende Idiozomformen; dasselbe Object und Stadium. Fig. 2 - eine deutliche Centralspindel: die Contur des Idiozoms an einer Stelle scharf abgebrochen. Fig. 3 - gelappte, unregelmulssige Form des Idiozoms mit zwei hantelförmigen Centralkörpern.

Fig. 4-8. Eier aus der Reifungsperiode - ausgetragenes Meerschweinchen.

Fig. 4. Junges Ei im beginnenden Spiremstadium. Idiozom mit Centralkörpern sehr scharf gegen das dichte Cytoplasma abgesetzt.

Fig. 5. Dasselbe Object: Spiremstadium: die Conturen des Idiozoms 
sehr undeutlich, zerfliessend, zwischen den Centrallsörper’ı eine kleine Centralspindel.

Fig. 6. Ein weiteres Mitosestadium: Kernmembran fast vollständig. aufgelöst. Das Chromatin in einzelne Schleifen mit regelmässiger Orientirung zerfallen. Die Contur des Idiozoms unregelmüssig zackig. $Z$ wischen den Centralkörpern eine Centralspindel.

Fig. 7. Die Chromatinschleifen zu einem Mutterstern geordnet. Die Centralspindel mit einer sehr deutlichen, von der Idiozomsubstanz ausgehenden Strahlung in einem höheren Niveau im Centrum des Sternes.

Fig. 8. Aequatorialplatte. An einem Pole schwache Andeutungen einer Polstrahlung. An den Spindelpolen geringe Anhäufungen einer sich rosaroth färbenden Substanz.

(Aus dem anatomischen Institut zu Freiburg i. Brsg.)

\section{Die Entwickelung der Milz bei den Amnioten.}

Von

\section{Dr. W. Tonkoff.}

Hierzu Tafel XVII, XVIII u. XIX und 8 Textfiguren.

Bekanntlich ist die Entwickelungsgeschichte der Milz im Verlaufe der letzten Jahrzelinte Gegenstand zahlreicher Untersuchungen gewesen. Die dabei gewonnenen Ergebnisse jedoch waren nichts weniger als übereinstimmend. Als Ursprungsstätteu sind alle nur möglichen Quellen in Betracht gezogen worden. Die Einen leiteten die Milz aus dem Mesenchym, die Andern aus dem Coelomepithel ab. Noch Andere liessen die Mily, analog den Drüsen des Darmkanales, sich durch Ausstülpung aus den Entoderm entwickeln. Nach Ansicht mancher Autoren soll die Milz aus Wanderzellen entodermalen Ursprunges hervorgehen. Auch eine gleichzeitige Betheiligung ron Entoderm, Mesoderm und Mesenchym oei der Bildung der Milz ist angenommen worden. Kurz als einzige Quelle, auf die bisher die Milz noch nicht 